\title{
DEPTOR has growth suppression activity against pancreatic cancer cells
}

\author{
Hua Li' ${ }^{1}$ Grace Y. Sun ${ }^{1}$, Yongchao Zhao ${ }^{1}$, Dafydd Thomas ${ }^{2}$, Joel K. Greenson ${ }^{2}$, \\ Mark M. Zalupski ${ }^{3}$, Edgar Ben-Josef ${ }^{1}$, Yi Sun ${ }^{1,4}$ \\ ${ }^{1}$ Division of Radiation and Cancer Biology, Department of Radiation Oncology, University of Michigan, Ann Arbor, MI 48109, USA \\ ${ }^{2}$ Department of Pathology, University of Michigan, Ann Arbor, MI 48109, USA \\ ${ }^{3}$ Department of Internal Medicine, University of Michigan, Ann Arbor, MI 48109, USA \\ ${ }^{4}$ Institute of Translational Medicine, Zhejiang University School of Medicine, Hangzhou, Zhejiang, P.R. China
}

Correspondence to:

Yi Sun, email: sunyi@umich.edu or yisun@zju.edu.cn

Keywords: DEPTOR, mTOR pathway, pancreatic cancer, growth suppression

Received: August 26, $2014 \quad$ Accepted: October 27, $2014 \quad$ Published: December 30, 2014

\section{ABSTRACT}

DEPTOR was reported as a naturally occurring inhibitor of mTORC1 and mTORC2. The role of DEPTOR in the growth and survival of pancreatic cancer cells has not previously been determined. Here we report that while DEPTOR shows a cytoplasmic expression in both normal pancreatic acinar and islet cells in a patchy manner, its expression is reduced in PanIN1 and PanIN2 and completely lost in 100 out of 101 pancreatic ductal adenocarcinoma (PDAC) tissues. Ectopic DEPTOR expression in two pancreatic cancer cell lines, Panc-1 and Miapaca-2, caused a significant 1) suppression of anchorage-dependent growth in monolayer culture, particularly under conditions with growth factor deprivation; 2) decreased clonogenic survival, and 3 ) suppressed anchorage-independent growth in soft agar. These effects are attributable to moderate induction of apoptosis and growth arrest at the S and G2/M phases, in a cell line dependent manner. Furthermore, ectopic DEPTOR expression moderately inhibited mTORC1 activity, as demonstrated by reduced phosphorylation of S6K, S6, and 4E-BP1. Taken together, these data suggest that DEPTOR has a tumor suppressive activity against pancreatic cancer cells, and its loss of expression may contribute to pancreatic tumorigenesis.

\section{INTRODUCTION}

DEPTOR is a recently identified mTOR binding protein that inhibits both mTORC1 and mTORC2 [1], the central regulators of cell metabolism, growth, proliferation, and survival [2-4]. In cell culture settings, DEPTOR mainly acts as a tumor suppressor, since DEPTOR loss activates mTORC1 and mTORC2 and promotes growth and survival of multiple cancer cell lines. However, in a subset of multiple myeloma cells, where DEPTOR was overexpressed, DEPTOR acts as an oncogene and survival factor, since DEPTOR inhibition of $\mathrm{mTORC} 1$ relieves the feedback inhibition from S6K1 to PI3K, boosting AKT activity for cancer cells survival $[1,2,5]$. We and others recently found that DEPTOR is a novel substrate of $\mathrm{SCF}^{\beta-\operatorname{TrCP}}(\mathrm{SKP} 1$, Cullins, F-box proteins) E3 ubiquitin ligase [6-8], the largest family of E3 ubiquitin ligases that regulates many biological processes via degrading the key regulators $[9,10]$. Upon growth factor exposure, DEPTOR was phosphorylated at the $\beta$-TrCP binding motif (D-pS-G-X-X-pS) on codons $286-291$ by RSK1/S6K1 kinases and then recognized by $\beta$-TrCP for targeted degradation by SCF E3, leading to mTOR activation [8]. We further showed that by degrading DEPTOR, $\mathrm{SCF}^{\beta-\operatorname{TrCP}}$ regulates cell survival [8].

Pancreatic ductal adenocarcinoma (PDAC) is the fourth leading cause of cancer death in the United States and amongst the deadliest human malignancies with an approximate 5 year survival of 5\% [11]. Mutational activation of the Kras oncogene occurs in $95 \%$ of cases, with inactivation of tumor suppressors, p53 and PTEN, and activation of PI3K-AKT-mTOR pathway occurring 
in $\sim 50-60 \%$ of cases [12-16]. In addition, NFאB, a downstream mediator of mutant Kras signaling in PDAC $[17,18]$, was found to be constitutively activated in most primary pancreatic cancers and cell lines [19, 20], due partly to the overexpression of $\beta$-TrCP [21], a F-box protein of SCF E3 ligase that promotes degradation of IKB [22] to activate NFKB and DEPTOR to activate mTORC1/2 [6-8].

Although DEPTOR has been shown to inhibit mTOR activity and may act as a tumor suppressor in some cell culture settings $[1,2]$, it has not been previously tested whether and how DEPTOR plays a role in pancreatic cancer. Here we show that DEPTOR expression is gradually reduced in pancreatic preneoplastic lesions, and completely lost in $99 \%$ of pancreatic ductal adenocarcinoma tissues. Ectopic expression of DEPTOR in pancreatic cancer cells suppressed their growth in monolayer culture and in soft agar, and decreased clonogenic survival by inducing apoptosis and growth arrest, likely through inactivation of mTORC1 signal. Our study suggests that DEPTOR acts as a tumor suppressor in pancreatic cancer and its loss may contribute to the initiation and progression of pancreatic tumorigenesis.

\section{RESULTS AND DISCUSSION}

\section{DEPTOR expression was lost in PDAC tumor tissues}

To determine potential role of DEPTOR during human pancreatic tumorigenesis, we determined the expression of DEPTOR protein in normal pancreas cells as well as in early pancreatic preneoplastic lesions and PDAC tissues. We found that DEPTOR is expressed in cytoplasm in both normal acinar (Figure 1A) and islet cells (Figure 1B) in a patchy manner. However, DEPTOR expression is gradually decreased from earlier-to-later stage of lesions, such as PanIN1 (Figure 1C), PanIN2 (Figure 1D), one PDAC tissue (Figure 1E). DEPTOR expression is completely lost in 100 out of 101 PDAC tissues (Figure 1F and data not shown). Thus, DEPTOR expression is gradually lost during human pancreatic tumorigenesis.

\section{Ectopic DEPTOR expression inhibits growth of pancreatic cancer cells in monolayer culture}

Given the observation that DEPTOR expression was lost in PDAC tissues, we next determined whether ectopic DEPTOR expression would affect the growth of pancreatic cancer cells. We transfected FLAG-tagged DEPTOR cDNA, along with the empty pcDNA3 control, into two commonly used pancreatic cancer lines, Panc1 and Miapaca-2. All resistant stable clones, after G418 selection, were pooled for Western blotting. As shown in Figure 2A, the levels of ectopically expressed DEPTOR were comparable to those of the endogenous DEPTOR in both Panc-1 and Miapaca-2 cells. We then measured the growth rate by ATP-lite proliferation assay for a 5-day period and found that in both cell lines, DEPTOR expression inhibited cell growth when cultured in normal medium containing 10\% fetal bovine serum (Figure 2B\&C). Growth suppression was more substantial if cells were cultured in serum starved condition with $1 \%$ FBS (Figure 2D\&E). Thus, DEPTOR expression had negative impact on the growth of pancreatic cancer cells, particularly under nutrient-deprived conditions.

\section{Ectopic DEPTOR expression inhibits clonogenic survival of pancreatic cancer cells}

We next used a standard clonogenic assay to measure the effect of DEPTOR expression on colony forming ability of these pancreatic cancer cells. Again, colony numbers formed from a single cell were significantly reduced upon ectopic DEPTOR expression in both lines (Figure 3A-D, with representative plates shown in $A \& B$ and quantitative data shown in C\&D). Thus, DEPTOR expression inhibits the clonogenic survival of pancreatic cancer cells.

\section{Ectopic DEPTOR expression inhibits anchorage- independent growth of pancreatic cancer cells}

We further determined the ability of DEPTORoverexpressing cells to grow in an anchorage independent manner in soft agar, which is a classic measurement of tumor cell phenotype. We found that again, DEPTOR expression significantly suppressed the formation of agar colonies (Figure 4A-D, with representative plates shown in $\mathrm{A} \& \mathrm{~B}$ and quantitative data shown in C\&D). Taken together, ectopic DEPTOR expression suppressed the growth of pancreatic cancer cells in all measures, indicating a tumor suppressive activity.

\section{Ectopic DEPTOR expression caused moderate induction of apoptosis and growth arrest}

We then determined the nature of growth suppression using FACS analysis. Cells were grown in monolayer culture for up to 3 days with samples harvested at every 24 hrs. FACS profile revealed that DEPTOR ectopic expression caused a moderate induction of sub-G1 apoptotic population in both cell lines, particularly at later time points (Figure 5A), and also caused growth arrest at the G2/M for Panc-1 cells and the S for Miapaca-2 cells (Figure 5B).

\section{Ectopic DEPTOR expression inhibits mTORC1 activity}

Finally, we evaluated potential mechanisms by which DEPTOR ectopic expression induced growth 

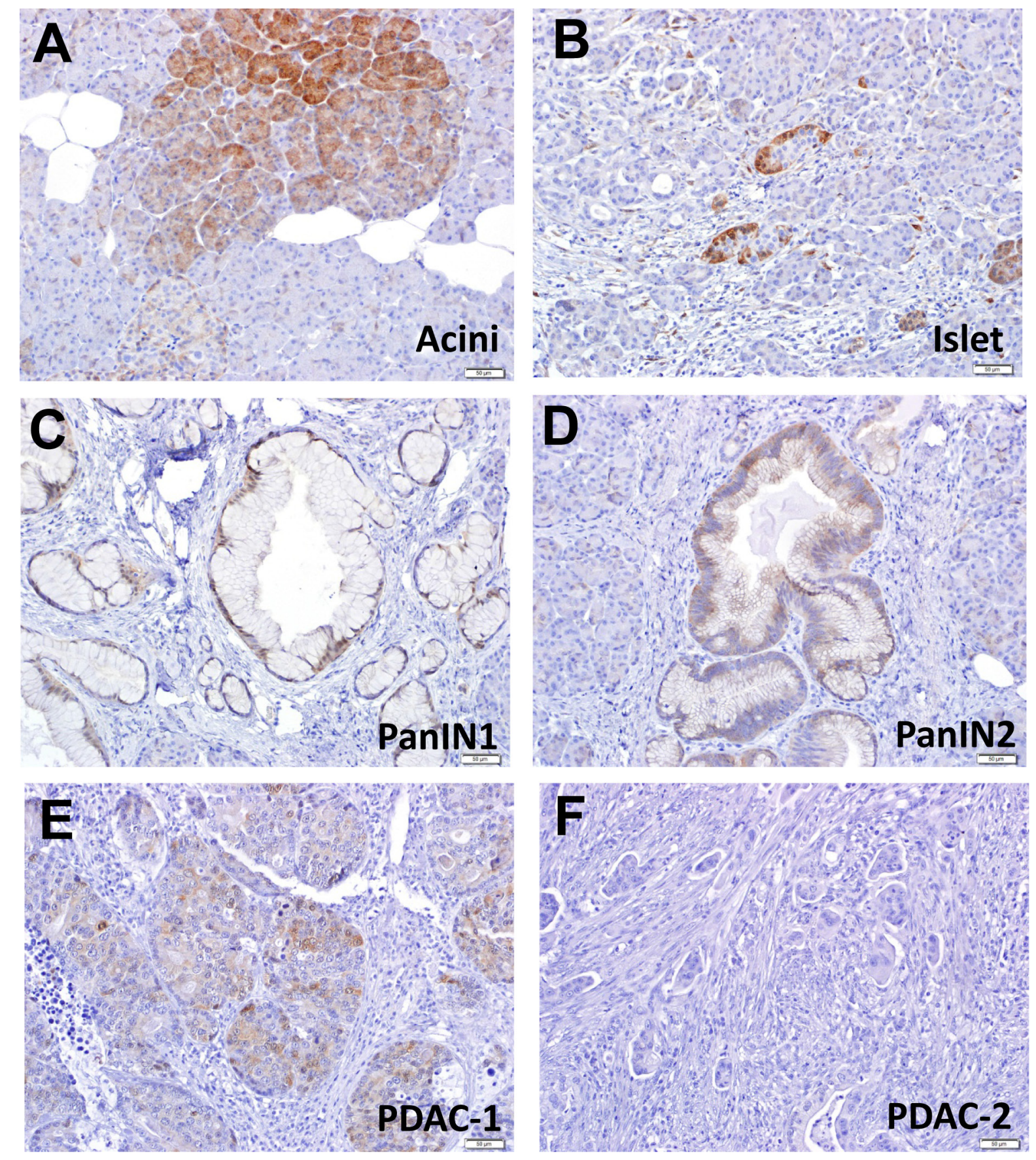

Figure 1: DEPTOR expression is gradually lost during human pancreatic tumorigenesis. two sets of PDAC tissue microarrays, containing a total of 101 PDAC tissues, along with 6 normal pancreas tissue slides, were stained with DEPTOR antibody. DEPTOR staining in all samples were scored in blind manner, and photographed. Normal pancreatic cells (A\&B), preneoplastic lesions (PanIN1, PanIN2) (C\&D), and PDAC (E\&F) were labeled as indicated.

suppression with a focus on mTORC1 and mTORC2. We found that $\mathrm{mTORC} 1$ activity was moderately inhibited upon DEPTOR ectopic expression, as reflected by reduced phosphorylation of mTORC1 substrate $\mathrm{S} 6 \mathrm{~K}$ and its substrate S6, as well as 4E-BP1, although to a lesser extent (Figure 6A). Interestingly, DEPTOR ectopic expression moderately increased the levels of phosphorylated AKT (Figure 6A), likely due to relieving the feedback inhibition from $\mathrm{S} 6 \mathrm{~K} 1$ to PI3K, as a result of mTORC1 inactivation $[1,2,5]$. However, DEPTOR ectopic expression had no effect on ERK signal (Figure 6A). Given the fact that growth rate was more severely affected when cells were 


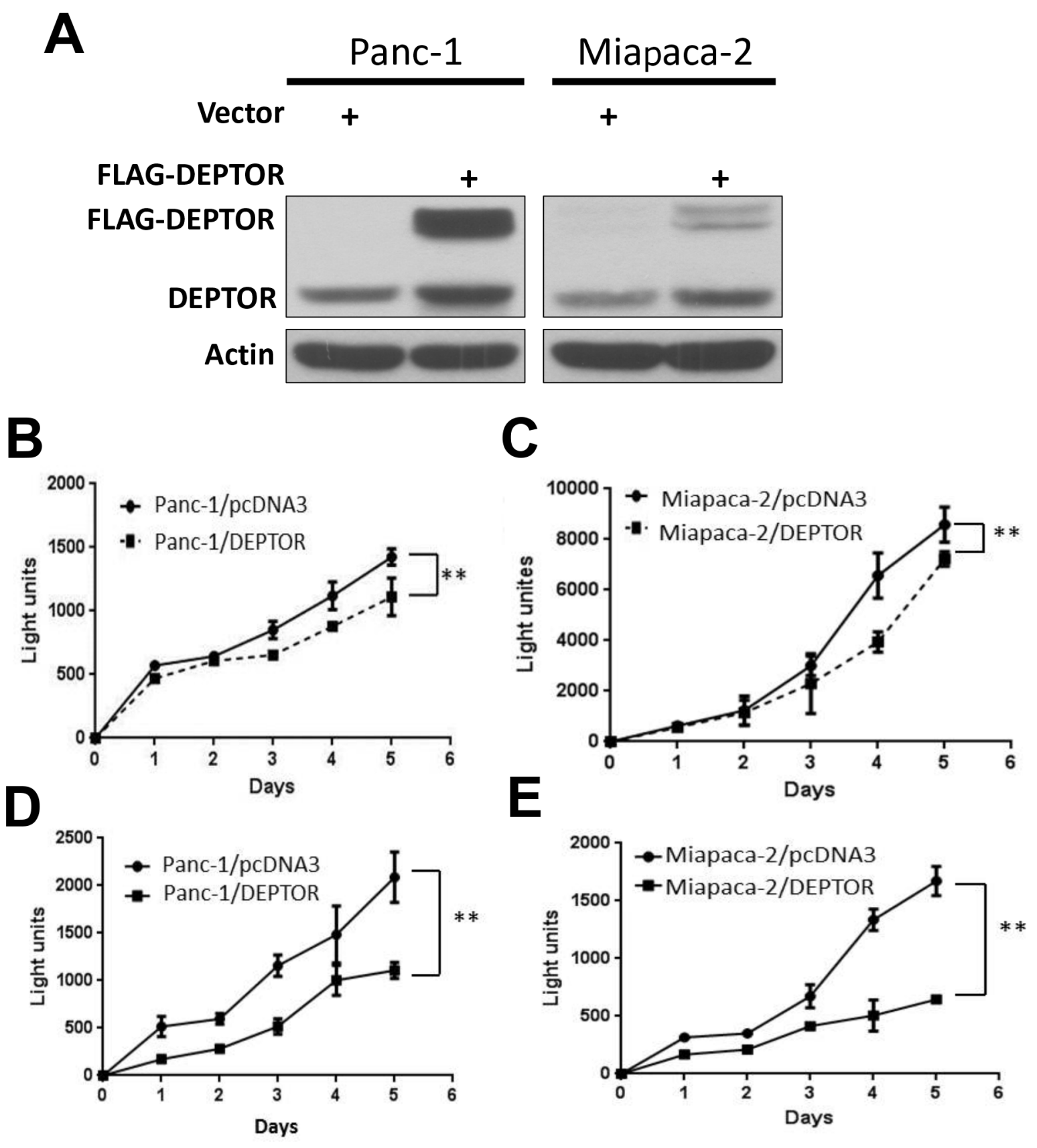

Figure 2: DEPTOR expression inhibits monolayer growth of PDAC cells. Panc-1 and Miapaca-2 cells were transfected with mammalian expressing vector encoding FLAG-DEPTOR, along with empty vector pcDNA3 control. Stable clones after G418 selection were pooled and subjected to IB using anti-DEPTOR antibody Ab (A). Cells were seeded and cultured in 96-well plates (1500/well) in triplicate for up to 5 days in DMEM with $10 \%$ FBS (B\&C) or DMEM with 1\% FBS (D\&E). Cells were lysed every 24 hrs and subjected to ATP-lite proliferation assay. Shown is $\mathrm{X} \pm \mathrm{SEM}$ of light unit $(\mathrm{n}=3)$. Student $t$ test was performed, $* *, p<0.01$.

cultured in $1 \%$ FBS condition in DEPTOR transfected cells (Figure 2B-E), we finally compared mTORC1 activity under growth conditions of $10 \%$ FBS vs. $1 \%$ FBS. While phosphorylation of S6K was undetectable when both lines of cells were cultured at 1\% FBS (data not shown), phosphorylation of 4E-BP1 was indeed more obviously inhibited (Figure 6B).
Several prior studies have reported conflicting correlations between DEPTOR expression and patient prognosis or disease status in human malignancies. One study showed that DEPTOR overexpression correlates with poor prognosis in differentiated thyroid carcinoma [23], whereas another study revealed that DEPTOR expression negatively correlates with tumor 


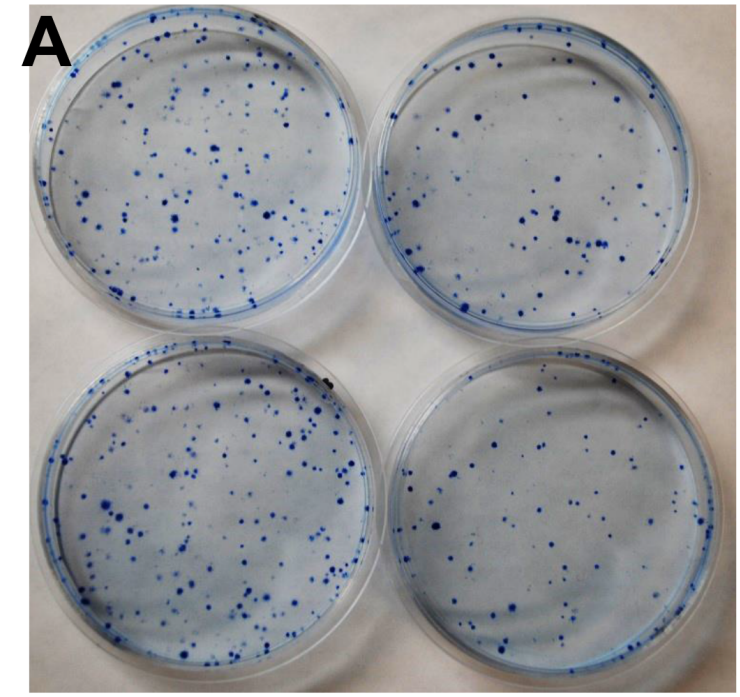

Panc-1/pcDNA3 Panc-1/DEPTOR
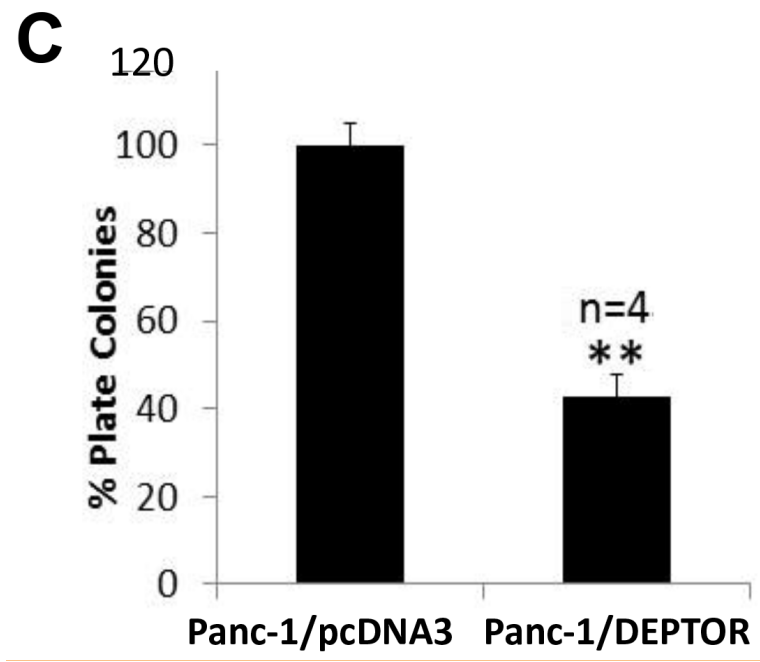

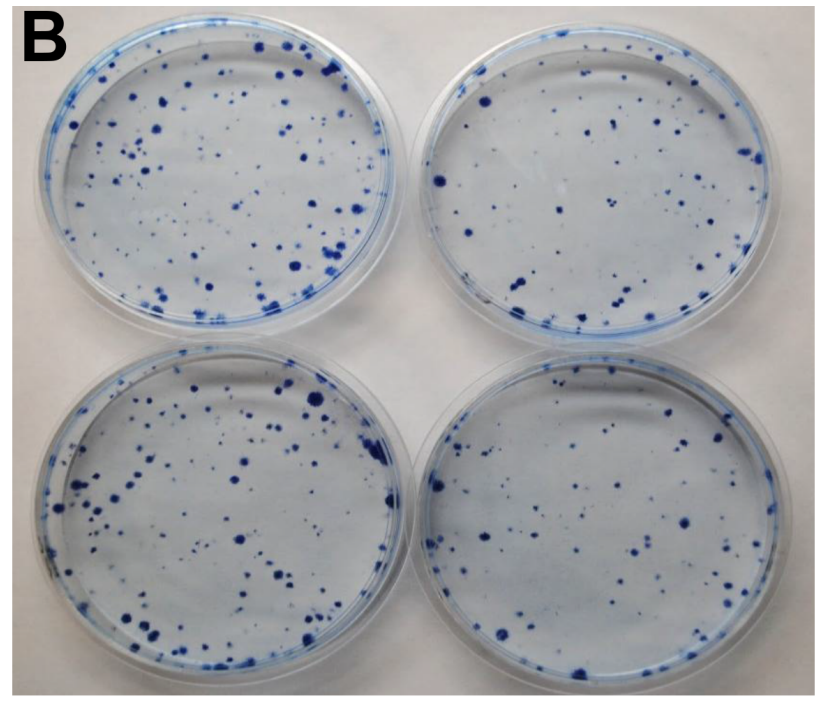

Miapaca-2/pcDNA3 Miapaca-2/DEPTOR

D

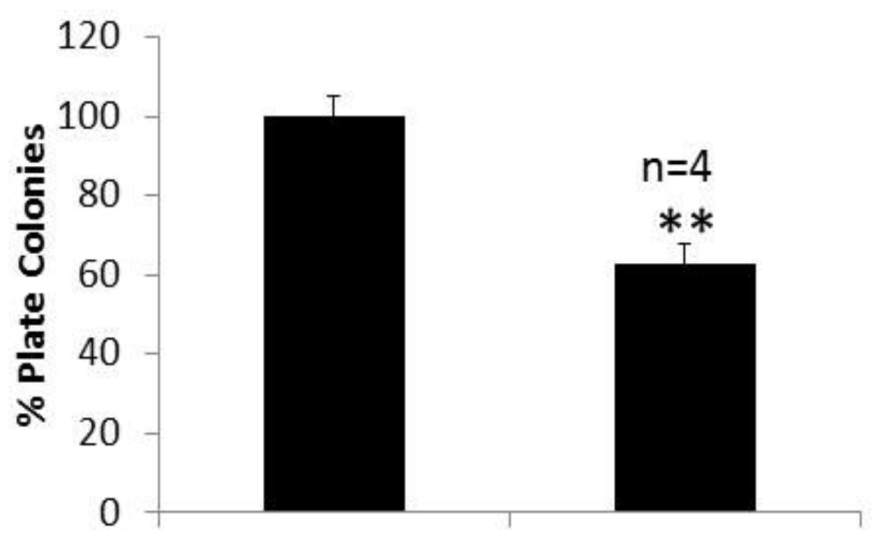

Miapaca-2/pcDNA3 Miapaca-2/DEPTOR

Figure 3: DEPTOR expression inhibits clonogenic survival of PDAC cells. Single cell suspension of Panc-1 and Miapaca-2 cells, stably expressing FLAG-DEPTOR were seeded, along with the vector control cells, into 60-mm dishes (600/dish) in DMEM with 10\% FBS and grown for 10-14 days. Colonies ( $\geq 50$ cells) were counted and plates were photographed (A\&B). Quantified results were expressed as percentage to the vector control, setting at $100 \%(\mathbf{C} \& \mathbf{D})$. Shown is $\mathrm{X} \pm \mathrm{SEM}$ of light unit $(\mathrm{n}=4)$. Student $t$ test was performed, **, $p<0.01$.

progression in colorectal cancer [24]. Here we show that DEPTOR expression is gradually lost during pancreatic tumorigenesis, and completely lost in PDAC tissues. We further showed that ectopic expression of DEPTOR at the level comparable to the endogenous one suppresses the growth of pancreatic cancer cells in monolayer culture, in clonogenic survival and in soft agar likely due to moderate induction of apoptosis and growth arrest at the $\mathrm{S}$ or $\mathrm{G} 2 / \mathrm{M}$ phases of cell cycle. Interestingly, we found that while DEPTOR overexpression moderately inactivates mTORC1, it activates PI3K/AKT as well, which was also seen in other model systems $[8,25]$. The fact that DEPTOR ectopic expression suppresses the growth of pancreatic cancer cells indicates that mTORC1 inactivation outcompetes AKT activation. Finally, it is noteworthy that growth suppression by ectopic DEPTOR expression may also be mediated by other yet-to-be identified mechanism(s) in addition to modulation of mTOR pathway. Taken together, our study provides experimental evidence that DEPTOR plays a tumor suppressive role in pancreatic cancer cells and loss of DEPTOR expression could contribute to pancreatic tumorigenesis. 


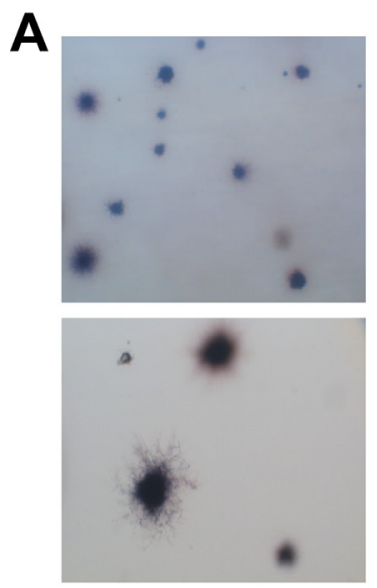

Panc-1/pcDNA3

C

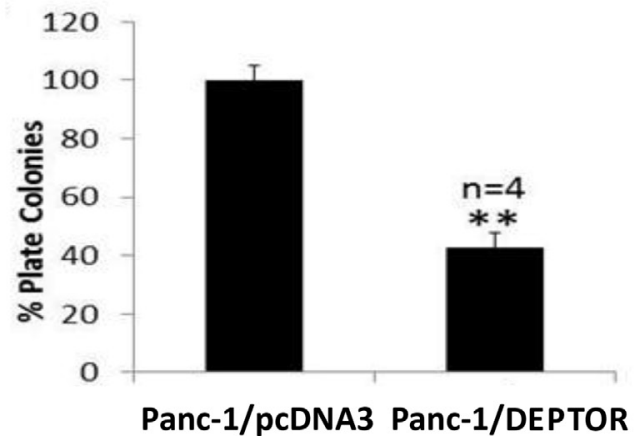

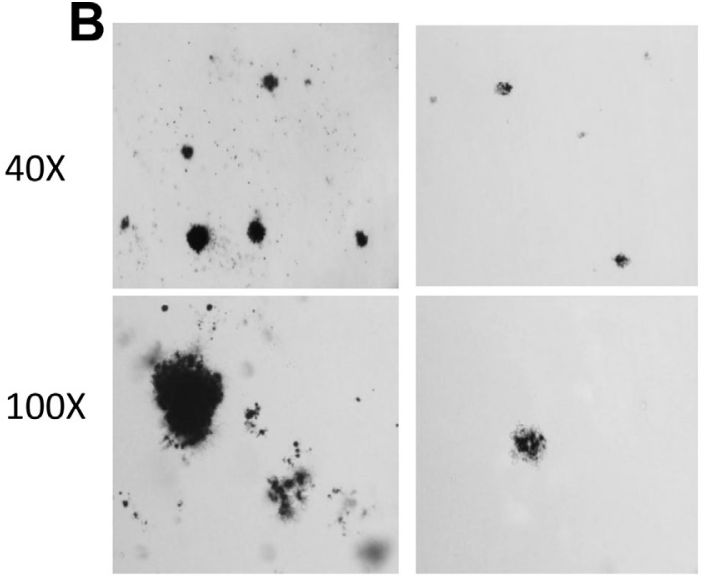

Miapaca-2/pcDNA3 Miapaca-2/DEPTOR

D

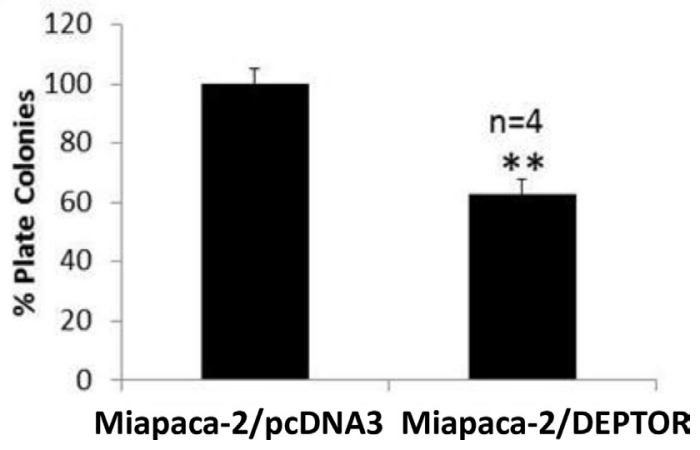

Figure 4: DEPTOR expression inhibits anchorage-independent growth of PDAC cells. Single cell suspension of Panc-1 and Miapaca- 2 cells, stably expressing FLAG-DEPTOR were seeded, along with the vector control cells, into 60 -mm agar dishes (1.5x104 cells/ dish), as described [31]. Colonies ( $\geq 8$ cells) were counted after 14 days and photographed (A\&B). Quantified results were expressed as percentage to the vector control, setting at $100 \%(\mathbf{C} \& D)$. Shown is $\mathrm{X} \pm \mathrm{SEM}(\mathrm{n}=4)$. Student $t$ test was performed, $* *, p<0.01$.
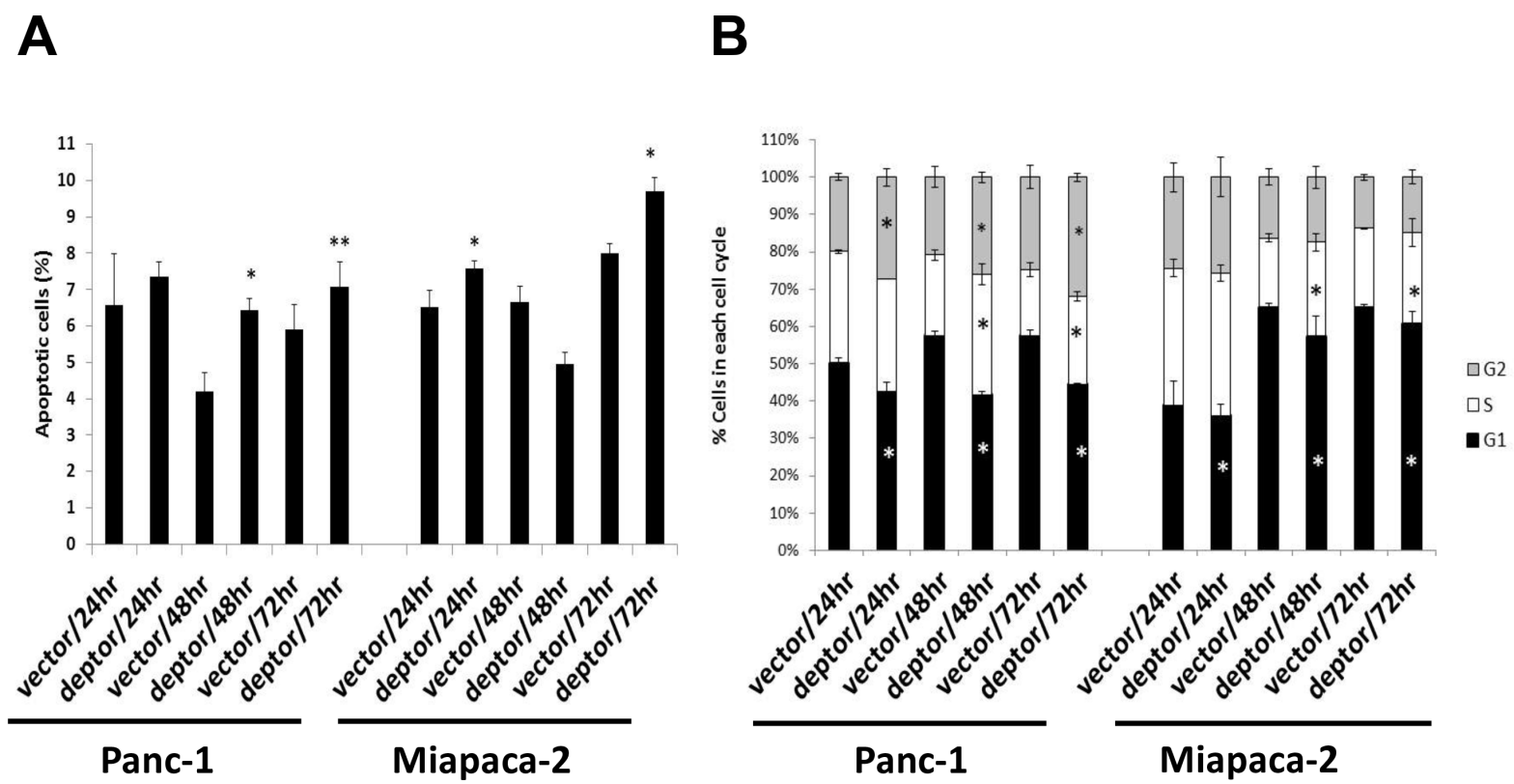

Figure 5: DEPTOR expression moderately induces apoptosis and growth arrest of PDAC cells. DEPTOR ectopically expressing Panc-1 and Miapaca- 2 cells were seeded, along with the vector control cells, into 60-mm dishes and cultured for up to $72 \mathrm{hrs}$ at DMEM with $10 \%$ FBS. Every $24 \mathrm{hrs}$ post seeding, cells were harvested and prepared for FACS analysis. Sub-G1 population was used to calculate \% of apoptotic cells (A). The remaining population was gated for each phase of cell cycle $(\mathbf{B})$. Shown is $\mathrm{X} \pm \operatorname{SEM}(\mathrm{n}=2)$. Student $t$ test was performed, ${ }^{*}, p<0.05$; $* *, p<0.01$. 


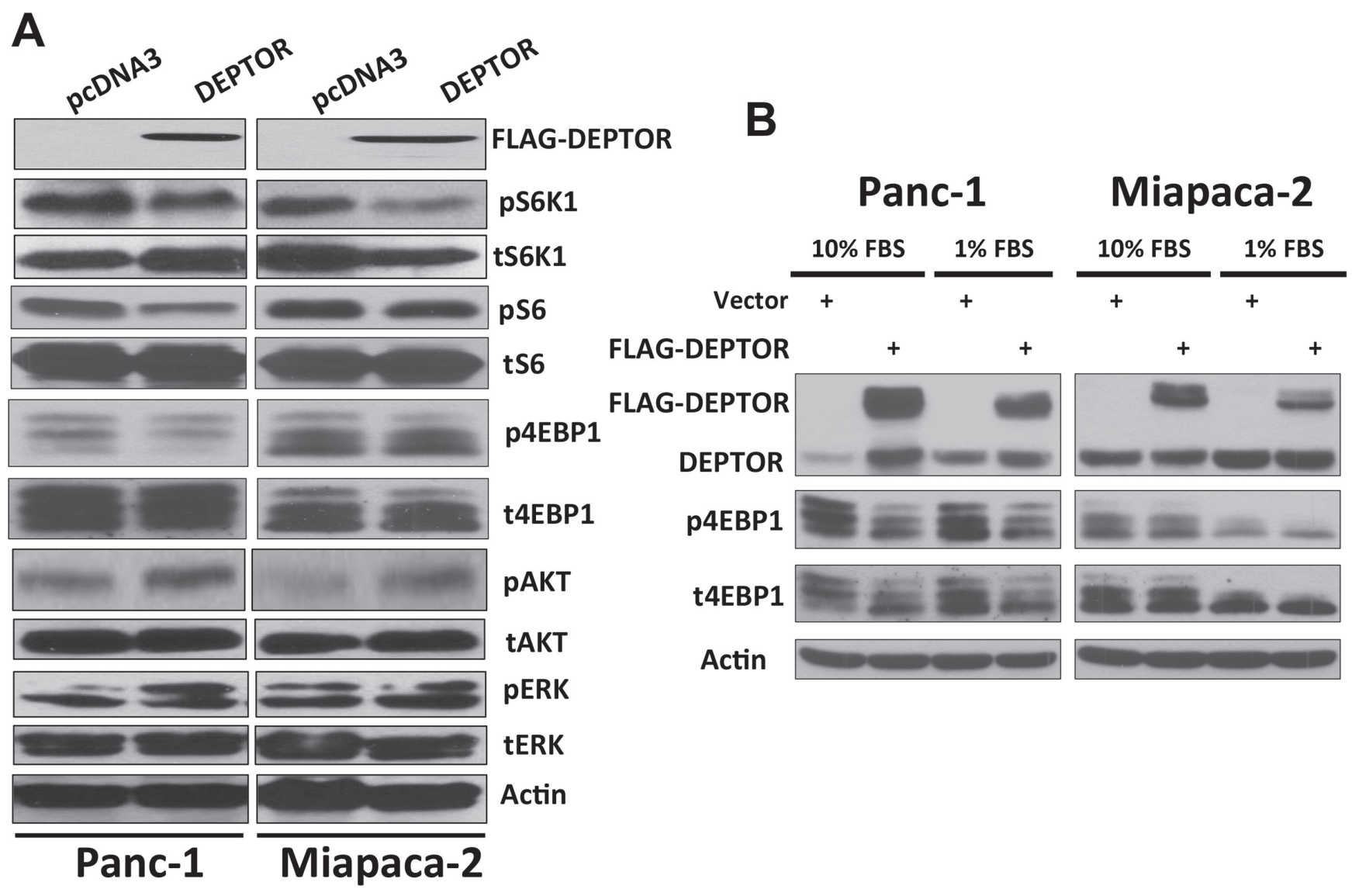

Figure 6: DEPTOR expression inhibits mTORC1 activity in PDAC cells. DEPTOR-ectopically expressing Panc-1 and Miapaca-2 cells were seeded, along with the vector control cells, into 60-mm dishes and cultured at DMEM with 10\%FBS (A) or 10\% v.s $1 \%$ FBS (B) for 24-48 hrs before being lysed for Western blotting analysis using indicated antibodies.

\section{METHODS}

\section{Cell culture and DNA Transfection}

Panc-1 and Miapaca-2 cells were cultured in DMEM supplemented with $10 \%$ or $1 \%$ fetal bovine serum. Cells were transfected with FLAG-DEPTOR, constructed in pcDNA3 [8], using Lipofactamine 2000. Following G418 selection of 2 weeks, resistant clones were pooled for Western blotting as described [26] for DEPTOR expression, and used for various growth assays. The antibodies were purchased as follows: DEPTOR, Phospho-4EBP1, 4EBP1, Phospho-ERK1/2 (Thr202/Tyr204), ERK1/2, PhosphoS6K1 (Thr389), Phospho-AKT (Ser473), AKT (Cell Signaling Technology, MA), S6K1, $\beta$-Actin (Santa Cruz Biotechnology, CA), FLAG (Sigma, MO).

\section{Immunohistochemical staining}

Collection and preparation of pancreatic cancer tissue microarrays containing 101 patient samples were partially described previously [27]. Immunohistochemical staining of the tissue microarrays, along with six normal pancreatic tissues, was performed using the $\mathrm{ABC}$ Vectastain kit (Vector Laboratories) with antibody against DEPTOR/ DEPDC6, as described [28]. Sections were developed with $\mathrm{DAB}$ and counterstained with haematoxylin.

\section{Cell growth assays}

For cell proliferation, an ATPlite kit (Perkin Elmer) was used as described [29] to measure growth rate within 5 days. For cell survival, a standard clonogenic assay was used as described [30]. For measurement of anchorage independent growth, a soft agar assay was used, as described [31].

\section{Fluorescence activated cell sorting (FACS)}

FACS analysis was performed as described [29]. Briefly, cells were harvested and fixed in $70 \% \mathrm{EtOH}$ at $-20^{\circ} \mathrm{C}$ for at least $4 \mathrm{~h}$, suspended in $1 \mathrm{X}$ Propidium Iodide solution with $400 \mathrm{mg} / \mathrm{ml}$ RNase (Roche), and analyzed in the Flow Cytometry Lab facility at the University of Michigan. The percent apoptosis is the percent of cells in the subG1 population. 


\section{ACKNOWLEDGEMENT}

This work is supported by the NCI grants (CA170995 and CA171277) to YS.

\section{REFERENCES}

1. Peterson TR, Laplante M, Thoreen CC, Sancak Y, Kang SA, Kuehl WM, Gray NS, Sabatini DM. DEPTOR is an mTOR inhibitor frequently overexpressed in multiple myeloma cells and required for their survival. Cell. 2009; 137:873-886.

2. Efeyan A, Sabatini DM. mTOR and cancer: many loops in one pathway. Curr Opin Cell Biol. 2010; 22:169-176.

3. Guertin DA, Sabatini DM. Defining the role of mTOR in cancer. Cancer Cell. 2007; 12:9-22.

4. Sabatini DM. mTOR and cancer: insights into a complex relationship. Nat Rev Cancer. 2006; 6:729-734.

5. Proud CG. Dynamic balancing: DEPTOR tips the scales. J Mol Cell Biol. 2009; 1:61-63.

6. Duan S, Skaar JR, Kuchay S, Toschi A, Kanarek N, Ben-Neriah Y, Pagano M. mTOR Generates an AutoAmplification Loop by Triggering the betaTrCP- and CK1alpha-Dependent Degradation of DEPTOR. Mol Cell. 2011; 44:317-324.

7. Gao D, Inuzuka H, Tan M-Kwang M, Fukushima H, Locasale Jason W, Liu P, Wan L, Zhai B, Chin YR, Shaik S. mTOR Drives Its Own Activation via SCF $\beta$ TrCPDependent Degradation of the mTOR Inhibitor DEPTOR. Molecular Cell. 2011; 44:290-303.

8. Zhao Y, Xiong X, Sun Y. DEPTOR, an mTOR Inhibitor, Is a Physiological Substrate of SCF $\beta$ TrCP E3 Ubiquitin Ligase and Regulates Survival and Autophagy. Molecular Cell. 2011; 44:304-316.

9. Deshaies RJ, Joazeiro CA. RING domain E3 ubiquitin ligases. Annu Rev Biochem. 2009; 78:399-434.

10. Jia L, Sun Y. SCF E3 ubiquitin ligases as anticancer targets. Curr Cancer Drug Targets. 2011; 11:347-356.

11. Jemal A, Bray F, Center MM, Ferlay J, Ward E, Forman D. Global cancer statistics. CA Cancer J Clin. 2011; 61:69-90.

12. Asano T, Yao Y, Zhu J, Li D, Abbruzzese JL, Reddy SA. The PI 3-kinase/Akt signaling pathway is activated due to aberrant Pten expression and targets transcription factors NF-kappaB and c-Myc in pancreatic cancer cells. Oncogene. 2004; 23:8571-8580.

13. Hezel AF, Kimmelman AC, Stanger BZ, Bardeesy N, Depinho RA. Genetics and biology of pancreatic ductal adenocarcinoma. Genes Dev. 2006; 20:1218-1249.

14. Matthaios D, Zarogoulidis P, Balgouranidou I, Chatzaki E, Kakolyris S. Molecular pathogenesis of pancreatic cancer and clinical perspectives. Oncology. 2011; 81:259-272.
15. Morris JPt Wang, SC Hebrok. KRAS, Hedgehog, Wnt and the twisted developmental biology of pancreatic ductal adenocarcinoma. Nat Rev Cancer. 2010; 10:683-695.

16. Ruggeri BA, Huang L, Wood M, Cheng JQ, Testa JR. Amplification and overexpression of the AKT2 oncogene in a subset of human pancreatic ductal adenocarcinomas. Molecular carcinogenesis. 1998; 21:81-86.

17. Sclabas GM, Fujioka S, Schmidt C, Evans DB, Chiao PJ. NF-kappaB in pancreatic cancer. Int J Gastrointest Cancer. 2003; 33:15-26.

18. Zhang Z, Rigas B. NF-kappaB, inflammation and pancreatic carcinogenesis: NF-kappaB as a chemoprevention target (review). Int J Oncol. 2006; 29:185-192.

19. Wang W, Abbruzzese JL, Evans DB, Larry L, Cleary KR, Chiao PJ. The nuclear factor-kappa B RelA transcription factor is constitutively activated in human pancreatic adenocarcinoma cells. Clin Cancer Res. 1999; 5:119-127.

20. Chandler NM, Canete JJ, Callery MP. Increased expression of NF-kappa B subunits in human pancreatic cancer cells. J Surg Res. 2004; 118:9-14.

21. Muerkoster S, Arlt A, Sipos B, Witt M, Grossmann M, Kloppel G, Kalthoff H, Folsch UR, Schafer H. Increased expression of the E3-ubiquitin ligase receptor subunit betaTRCP1 relates to constitutive nuclear factor-kappaB activation and chemoresistance in pancreatic carcinoma cells. Cancer Res. 2005; 65:1316-1324.

22. Winston JT, Strack P, Beer-Romero P, Chu CY, Elledge SJ, Harper JW. The SCFbeta-TRCP-ubiquitin ligase complex associates specifically with phosphorylated destruction motifs in IkappaBalpha and beta-catenin and stimulates IkappaBalpha ubiquitination in vitro. Genes Dev. 1999; 13:270-283.

23. Pei L, Xie P, Zhou E, Yang Q, Luo Y, Tang Z. Overexpression of DEP domain containing mTOR-interacting protein correlates with poor prognosis in differentiated thyroid carcinoma. Molecular medicine reports. 2011; 4:817-823.

24. Lai EY, Chen ZG, Zhou X, Fan XR, Wang H, Lai PL, $\mathrm{Su}$ YC, Zhang BY, Bai XC, Li YF. DEPTOR Expression Negatively Correlates with mTORC1 Activity and Tumor Progression in Colorectal Cancer. Asian Pacific journal of cancer prevention : APJCP. 2014; 15:4589-4594.

25. Meng ZX, Li S, Wang L, Ko HJ, Lee Y, Jung DY, Okutsu M, Yan Z, Kim JK, Lin JD. Baf60c drives glycolytic metabolism in the muscle and improves systemic glucose homeostasis through Deptor-mediated Akt activation. Nat Med. 2013; 19:640-645.

26. Sun SH, Zheng M, Ding K, Wang S, Sun Y. A small molecule that disrupts Mdm2-p53 binding activates p53, induces apoptosis, and sensitizes lung cancer cells to chemotherapy. Cancer Biol Ther. 2008; 7:845-852.

27. Desai S, Ben-Josef E, Griffith KA, Simeone D, Greenson JK, Francis IR, Hampton J, Colletti L, Chang AE, Lawrence TS. 
Gemcitabine-based combination chemotherapy followed by radiation with capecitabine as adjuvant therapy for resected pancreas cancer. International journal of radiation oncology, biology, physics. 2009; 75:1450-1455.

28. Li H, Tan M, Jia L, Wei D, Zhao Y, Chen G, Xu J, Zhao L, Thomas D, Beer DG. Inactivation of SAG/RBX2 E3 ubiquitin ligase suppresses KrasG12D-driven lung tumorigenesis. J Clin Invest. 2014; 124:835-846.

29. Bockbrader KM, Tan M, Sun Y. A small molecule Smacmimic compound induces apoptosis and sensitizes TRAILand etoposide-induced apoptosis in breast cancer cells. Oncogene. 2005; 24:7381-7388.
30. Zheng M, Morgan-Lappe SE, Yang J, Bockbrader KM, Pamarthy D, Thomas D, Fesik SW, Sun Y. Growth inhibition and radiosensitization of glioblastoma and lung cancer cells by small interfering RNA silencing of tumor necrosis factor receptor-associated factor 2. Cancer Res. 2008; 68:7570-7578.

31. Gu Q, Tan M, Sun Y. SAG/ROC2/Rbx2 is a novel activator protein-1 target that promotes c-Jun degradation and inhibits 12-O-tetradecanoylphorbol-13-acetate-induced neoplastic transformation. Cancer Res. 2007; 67:3616-3625. 